\title{
Recording of cardiovascular risk factors by general practitioners in patients with schizophrenia
}

\author{
Ma Carmen Castillejos ${ }^{1^{*}}$ (D) , Carlos Martín-Pérez ${ }^{2}$, Antonio García-Ruiz ${ }^{3}$, Fermín Mayoral-Cleries ${ }^{4}$ \\ and Berta Moreno-Küstner ${ }^{5}$
}

\begin{abstract}
Background: Patients with schizophrenia and related disorders (SRD) are more predisposed to having cardiovascular risk factors (CVRFs) compared to the general population due to a combination of lifestyle factors and exposure to antipsychotic medications. We aimed to analyse the documentation practices of CVRFs by general practitioners (GPs) and its associations with patient variables in a sample of persons with SRD.

Methods: An observational, cross-sectional study was conducted in 13 primary care centres (PCCs) in Malaga (Spain). The population comprised all patients with SRD who were in contact with a GP residing in the study area. The number of CVRFs (type 2 diabetes mellitus, hypertension, hypercholesterolaemia, obesity and smoking) recorded by GPs were analysed by considering patients' demographic and clinical variables and use of primary care services. We performed descriptive, bivariate and multivariate regression analyses.

Results: A total of 494 patients were included; CVRFs were not recorded in 59.7\% of the patients. One CVRF was recorded in $42.1 \%$ of patients and two or more CVRFs were recorded in $16.1 \%$ of patients. Older age, living in an urban area and a higher number of visits to the GP were associated with a higher number of CVRFs recorded.
\end{abstract}

Conclusion: The main finding in this study is that both patients' demographic variables as well as use of primary care services were found to be related to the documentation of CVRFs in patients with SRD by GPs.

Keywords: Cardiovascular disorders, Medical comorbidity, Mental health, Primary care, Severe mental illness

\section{Background}

It has long been accepted that patients with severe mental illness (SMI) have higher rates of mortality and morbidity from physical health problems compared to the general population [1-3], due to the high level of CVRFs they present [4]. A meta-analysis performed by Osborn et al. [5] revealed that these patients presented a pooled risk ratio of 1.70 (95\% confidence interval [CI] 1.21-2.37) for

\footnotetext{
*Correspondence: mccasang@gmail.com

${ }^{1}$ Department of Personality, Assessment and Psychological Treatment,

Faculty of Psychology Andalusian Group of Psychosocial Research (GAP),

University of Malaga, Campus Teatinos, 29071 Malaga, Spain

Full list of author information is available at the end of the article
}

diabetes and 1.11 (95\% CI 0.91-1.35) for hypertension. In a further study carried out in London [5], patients with schizophrenia exhibited greater risk of developing CVRF, such as diabetes, hypertension, hyperlipidaemia, smoking and obesity. These results are congruent with many other studies [6-11].

Nevertheless, little attention continues to be paid to the physical problems of patients with SMI, especially considering that these individuals are at risk of underdiagnosis and under-treatment [12]. Smith et al. [3] reported that most people with schizophrenia had at least one chronic physical comorbidity and one-third had two or more. However, cardiovascular disorder comorbidities among these patients were under-recorded relative

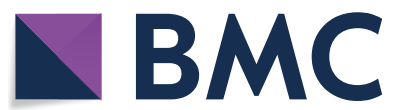

(c) The Author(s) 2020. This article is licensed under a Creative Commons Attribution 4.0 International License, which permits use, sharing, adaptation, distribution and reproduction in any medium or format, as long as you give appropriate credit to the original author(s) and the source, provide a link to the Creative Commons licence, and indicate if changes were made. The images or other third party material in this article are included in the article's Creative Commons licence, unless indicated otherwise in a credit line to the material. If material is not included in the article's Creative Commons licence and your intended use is not permitted by statutory regulation or exceeds the permitted use, you will need to obtain permission directly from the copyright holder. To view a copy of this licence, visit http://creativeco mmons.org/licenses/by/4.0/. The Creative Commons Public Domain Dedication waiver (http://creativecommons.org/publicdomain/ zero/1.0/) applies to the data made available in this article, unless otherwise stated in a credit line to the data. 
to individuals without schizophrenia. Woodhead et al. [13] found similar results, notwithstanding the fact that the number of consultations with primary care was high. Additionally, patients with SMI receive fewer prescriptions for the treatment of common CVRFs [14]. This phenomenon could be because most health professionals consider the care of people with SMI too specialised for primary care [15].

Patients with SRD are more predisposed to having CVRFs compared to the general population due to a combination of lifestyle factors and exposure to antipsychotic medications. We hypothesise that there may be a relationship between the number of CVRFs recorded in the clinical records of patients with SRD by GPs and patients' demographic and clinical variables and use of primary care services variables. We aimed to examine the documentation practices of CVRFs (type 2 diabetes mellitus, hypertension, hypercholesterolaemia, obesity and smoking) by GPs in patients with SRD and their association with patients' demographic and clinical variables and use of primary care services.

\section{Methods}

An observational, cross-sectional study was conducted.

\section{Setting of the study}

This study was implemented in the area of the Clinical Management Unit of Mental Health (CMU-MH) of the Regional Hospital of Malaga, whose population coverage comprises 315,159 inhabitants. This area includes two community mental health units (CMHU: Centre and North), which contain 13 primary care centres (PCCs). The study information was collected from 1 January, 2008 to 1 July, 2011.

\section{Patient and public involvement}

The Malaga Schizophrenia Case Registry (RESMA) is a case registry of patients with SRD who attend the CMU$\mathrm{MH}$ of the Regional Hospital of Malaga to improve the care of patients with SMI [16].

The inclusion criteria were patients: (a) aged 15 and older; (b) with a clinical diagnosis of SRD according to the International Classification of Diseases (ICD-10); and (c) in contact with a PCC in the coverage area of the CMU-MH of the Regional Hospital of Malaga. The exclusion criteria were patients: a) treated outside the study area; b) who died during the follow-up; and c) not having a computerised medical record at the reference PCC [17].

The eligible population comprised all patients included in the RESMA $(N=1663)$ [18], from which we selected via simple random sampling a representative sample of all PCCs. A total of 528 patients were selected, but 34 (6\%) did not have a digitalised medical record in primary care, and so were not included in the study. Ultimately, a total of 494 patients were included.

\section{Study variables}

Our dependent variable was the number of CVRFs (type 2 diabetes mellitus, hypertension, hypercholesterolaemia, obesity and smoking) recorded by the SRD patients' GPs. The independent variables were: (1) demographic factors: gender (male or female); age (15-44, 45-64 or > 65 years); marital status (single, married/civil partnership/cohabiting or separated/divorced/widowed); educational level (no formal education and/or illiterate, primary school, secondary school or higher education [bachelor's degree or higher]); living arrangements (alone, living with parents/other relatives or friends, own family, sheltered accommodation or homeless); employment status (employed, unemployed, student, carer or househusband/housewife, receiving welfare benefits or temporary work disability); type of living area (urban or rural); living within a socioeconomically deprived area (no or yes); (2) clinical factors: ICD-10 clinical diagnosis (F20 schizophrenia, F22 persistent delusional disorders, F23 acute and transient psychotic disorders, F25 schizoaffective disorders, F21 schizotypal disorder, F24 induced delusional disorder, F28 other non-organic psychotic disorders or F29 unspecified non-organic psychosis); Global Level of Severity (GLS; level I: low severity, level II: medium severity, level III: high severity); and (3) use of primary care services: number of visits to GP; number of visits to nurse; total number of visits to PCC (including GPs and nurses).

The GLS is an index assigned to patients with SRD by psychiatrists, who usually treat patients according to symptom severity, disease evolution, social adjustment, disability level and treatment adherence. This index is used by psychiatrists in the clinical setting of public mental health services in order to include patients within the category of "Severe Mental Illness" and thus access additional resources [19].

\section{Data sources}

Data on patients' number of CVRFs and visits to their GP, nurse and PCC were obtained from digitised primary care records (DIRAYA programme). Patients' demographic and clinical information were collected from the RESMA [16, 18].

\section{Data analysis}

The categorical variables used in the descriptive analysis were reported using frequency distribution and percentages. Descriptive statistics (mean, standard deviation, median and quantiles) were calculated for the continuous variables. 
We used bivariate analysis to examine the relationships between the dependent variable (CVRFs) and the independent variables. To study the association between the dependent variable and the categorical explanatory variables with two categories, a non-parametric test (Wilcoxon) was used. In the case of a categorical explanatory variable that was continuous or had more than two categories, we used simple linear regression.

Finally, a linear regression was adjusted. In the first step, an initial model was adjusted with all the explanatory variables. In the second step, the variables that did not reach statistical significance were removed from the model; this did not imply substantial changes in the coefficients of the remainder of the variables. Differences were considered significant at $p<0.05$.

\section{Results}

The database comprised 494 patient records, of which $70 \%$ were men. The mean age at the beginning of the study was 43.8 years (SD 11.64). The majority of patients were single (70.6\%). Most of the patients had not completed secondary school (64.4\%). The most common living arrangements comprised living with parents, other relatives or friends (56.9\%). As regards patients' work situations, $42.9 \%$ were unable to work. Patients predominantly lived in an urban environment (87.1\%). Only $11.5 \%$ of the sample resided in socioeconomically deprived areas (Table 1).

The most frequent diagnosis was schizophrenia (70.04\%), followed by persistent delusional disorders (10.7\%), schizoaffective disorder (9.3\%), acute and transient psychotic disorders (7.3\%) and, finally, $2.6 \%$ of patients presented other diagnoses. More than a third of patients presented level II severity (38.2\%; Table 1 ).

During the study period, the mean number of patients' visits to their PCC was 22.25 (SD 21.32; 0-100), mean number of visits to their GP was 14.35 (SD 12.45; 5-75) and mean number of visits to their nurse was 7.75 (SD 14.89; 2-93; Table 2).

Regarding patients' number of recorded CVRFs, $59.7 \%$ did not present any recorded CVRFs, $42.1 \%$ presented one recorded CVRF and $16.1 \%$ presented two or more. Type 2 diabetes mellitus was recorded in $10.5 \%$ of patients, hypertension in $13.2 \%$, dyslipidaemia in $15.8 \%$ and obesity in $10.3 \% ; 14.2 \%$ were smokers.

\section{Factors associated with the number of documented CVRFs} The bivariate analysis indicated that the factors associated with a lower number of recorded CVRFs were: being male $(p=0.028)$, residing in rural areas $(p=0.005)$, having secondary $(p=0.022)$ or higher $(p=0.015)$ levels of education and being employed $(p=0.004)$, unemployed $(p<0.001)$, students $(p=0.012)$ or on temporary work disability $(p<0.001)$ compared with receiving welfare benefits. On the other hand, the factors that were associated with a greater number of recorded CVRFs were: having a partner $(p=0.002)$, being older $(p<0.001)$, residing in socioeconomically deprived areas $(p=0.048)$, presenting a level I illness severity $(p=0.030)$ and increased number of visits to the GP, nurse and PCC $(p<0.001)$. There were no significant differences in the type of living arrangement and diagnosis categories (Table 2).

The multivariate linear model revealed that there were five variables that presented a clear association with the number of CVRFs recorded by GPs: age, living arrangements, type of living area, employment status and number of visits to the PCC. Older age was associated with a greater number of recorded CVRFs $(p<0.001)$. Patients who had their own family $(p=0.049)$ and those who lived in sheltered accommodation $(p=0.048)$ presented more recorded CVRFs compared with homeless patients. The higher the number of patient's visits to the PCC, the greater the number of CVRFs recorded $(p<0.001)$. On the other hand, fewer CVRFs were recorded in patients living in rural areas $(p=0.028)$. For the employment category, only the group classified as temporary work disability $(p=0.022)$ had fewer CVRFs recorded compared to those who were receiving welfare benefits (Table 3).

\section{Discussion}

This study examined the recording practices by GPs of CVRFs of patients with SRD. The novelty of this study is that we were able to analyse a large number of patients with SRD in a primary care setting.

The percentages of CVRFs recorded in our sample were lower than those obtained for the general Andalusian population [20]. This result is unexpected given that the scientific literature describes SRD patients as being more predisposed to having multiple CVRFs [4]. Interestingly, similar results to the current study were reported in a Spanish study by Viñas et al. [21]. In Spain, all patients are assigned a GP, who represents the gateway to the Spanish health system and can refer patients to specialists. Thus, all patients are first treated by their GP, who refers them to a psychiatrist or other specialist if and when necessary. Viñas et al. explained their results as owing to these patients having limited access to the health system, hence they could not benefit from the preventative initiatives offered to the general population. Additionally, there was a lack of coordination between GPs and psychiatrists in patient care [21]. Bernardo et al. [22] found that factors such as smoking, alcoholism and excess weight were easily recognisable by doctors. However, factors such as dyslipidaemia were underdiagnosed, and a sedentary lifestyle was not recognised as a risk factor. Further studies may be necessary in this area for a 
Table 1 Sociodemographic and clinical characteristics of patients with schizophrenia and related disorders $(N=494)$

Sociodemographic characteristics

Gender

Male

Female

Age

15-44

45-64

$>65$

Marital status

single

Married/civil partnership/cohabiting

Separated/divorced/widowed

Educational level

No formal education and/or illiterate

Primary school

Secondary school

Higher education (bachelor's degree)

Living arrangements

Alone

With parents/other relatives or friends

Own family

Sheltered accommodation

Homeless

Employment status

Employed

Unemployed

Student

Carer or househusband/housewife

Receiving welfare benefits

Temporary work disability

Type of living area

Urban

Rural

Living within a socioeconomically deprived area

No

Yes

Clinical characteristics

ICD-10 clinical diagnosis

F20 Schizophrenia

$346(70 \%)$

F22 Persistent delusional disorders

$53(10.7 \%)$

F23 Acute and transient psychotic disorders

$46(7.3 \%)$

$36(9.3 \%)$

F25 Schizoaffective disorders

$13(2.6 \%)$

21, F24, F28, F29 Schizotypal disorder, Induced delusional disorder, other non-organic psychotic disorders and unspecified nonorganic psychosis

Global level of severity

Level I (low severity)

$157(31.8 \%)$

Level II

$189(38.2 \%)$

$85(17.2 \%)$

Level III (high severity) (Missing data:63)

$494(100 \%)$ 
Table 2 Bivariate analysis of the number of cardiovascular risk factors recorded in patients with schizophrenia and related disorders $(N=494)$

\begin{tabular}{|c|c|c|c|c|c|}
\hline & W Wilconxon & Estimate & Standar error & $t$ value & $p$ value \\
\hline Gender & 30202.5 & & & & 0.028 \\
\hline \multicolumn{6}{|l|}{ Male } \\
\hline \multicolumn{6}{|l|}{ Female } \\
\hline Age & & 0.024 & 0.004 & 6.757 & $p<0.001$ \\
\hline \multicolumn{6}{|l|}{ Marital status } \\
\hline Single & & Reference & & & \\
\hline Married/Civil partnership/Cohabiting & & 0.339 & 0.111 & 3.055 & 0.002 \\
\hline Separated/Divorced/Widowed & & 0.250 & 0.136 & 1.836 & 0.067 \\
\hline \multicolumn{6}{|l|}{ Educational level } \\
\hline No formal education and/or illiterate & & Reference & & & \\
\hline Primary school & & -0.114 & 0.121 & -0.945 & 0.345 \\
\hline Secondary school & & -0.307 & 0.134 & -2.291 & 0.022 \\
\hline Higher education (Bachelor's degree) & & -0.415 & 0.169 & -2.452 & 0.015 \\
\hline \multicolumn{6}{|l|}{ Living arrangements } \\
\hline Homeless & & Reference & & & \\
\hline Alone & & 0.522 & 0.439 & 1.189 & 0.235 \\
\hline With parents / other relatives or friends & & 0.330 & 0.424 & 0.779 & 0.436 \\
\hline Own family & & 0.643 & 0.430 & 1.494 & 0.136 \\
\hline Sheltered accommodation & & 0.588 & 0.440 & 1.337 & 0.182 \\
\hline \multicolumn{6}{|l|}{ Employment status } \\
\hline Receiving welfare benefits & & Reference & & & \\
\hline Employed & & -0.358 & 0.124 & -2.889 & 0.004 \\
\hline Unemployed & & -0.421 & 0.124 & -3.541 & $p<0.001$ \\
\hline Student & & -0.465 & 0.183 & -2.537 & 0.012 \\
\hline Carer or househusband/housewife & & 0.122 & 0.181 & 0.678 & 0.498 \\
\hline Temporary work disability & & -0.457 & 0.134 & -3.421 & $p<0.001$ \\
\hline Type of area & 16388.5 & & & & 0.005 \\
\hline \multicolumn{6}{|l|}{ Urban } \\
\hline \multicolumn{6}{|l|}{ Rural } \\
\hline Living within a socioeconomically deprived area & 10690 & & & & 0.048 \\
\hline \multicolumn{6}{|l|}{ Yes } \\
\hline \multicolumn{6}{|l|}{ No } \\
\hline \multicolumn{6}{|l|}{ ICD-10 clinical diagnosis } \\
\hline F20 schizophrenia & & Reference & & & \\
\hline $\begin{array}{l}\text { F21, F24, F28, F29 schizotypal disorder, induced delusional disorder, other } \\
\text { non-organic psychotic disorders and unspecified non-organic psychosis }\end{array}$ & & -0.274 & 0.268 & -1.024 & 0.306 \\
\hline F22 persistent delusional disorders & & -0.036 & 0.140 & -0.260 & 0.795 \\
\hline F23 acute and transient psychotic disorders & & -0.159 & 0.149 & -1.068 & 0.286 \\
\hline F25 schizoaffective disorders & & 0.091 & 0.166 & 0.548 & 0.584 \\
\hline \multicolumn{6}{|l|}{ Global level of severity } \\
\hline Level III (high severity) & & Reference & & & \\
\hline Level II & & 0.183 & 0.126 & 1.455 & 0.147 \\
\hline Level I (low severity) & & 0.283 & 0.130 & 2.180 & 0.030 \\
\hline Number of visits to primary care centre & & 0.015 & 0.002 & 8.129 & $p<0.001$ \\
\hline Number of visits to general practitioner & & 0.026 & 0.003 & 8.140 & $p<0.001$ \\
\hline Number of visits to nurse & & 0.013 & 0.003 & 4.660 & $p<0.001$ \\
\hline
\end{tabular}


Table 3 Multilevel linear regression of the number of cardiovascular risk factors recorded in patients with schizophrenia and related disorders $(=494)$

\begin{tabular}{|c|c|c|c|c|}
\hline & Estimate & Standar Error & $t$ value & $p$ value \\
\hline Age & 0.017 & 0.004 & 4.474 & $p<0.001$ \\
\hline \multicolumn{5}{|l|}{ Living arrangements } \\
\hline Homeless & Reference & & & \\
\hline Alone & 0.720 & 0.402 & 1.791 & 0.074 \\
\hline With parents/other relatives or friends & 0.706 & 0.390 & 1.812 & 0.071 \\
\hline Own family & 0.781 & 0.395 & 1.975 & 0.049 \\
\hline Sheltered accommodation & 0.798 & 0.403 & 1.983 & 0.048 \\
\hline \multicolumn{5}{|l|}{ Employment status } \\
\hline Receiving welfare benefits & Reference & & & \\
\hline Employed & -0.167 & 0.121 & -0.606 & 0.168 \\
\hline Unemployed & -0.172 & 0.117 & -1.476 & 0.141 \\
\hline Student & -0.108 & 0.178 & -0.606 & 0.545 \\
\hline Carer or househusband/housewife & 0.029 & 0.172 & 0.165 & 0.869 \\
\hline Temporary work disability & -0.290 & 0.126 & -2.302 & 0.022 \\
\hline \multicolumn{5}{|l|}{ Type of living area } \\
\hline Urban & Reference & & & \\
\hline Rural & -0.255 & 0.116 & -2.200 & 0.028 \\
\hline Number of visits to primary care centre & 0.013 & 0.002 & 7.204 & $p<0.001$ \\
\hline
\end{tabular}

reliable comparison of the documentation of CVRFs in patients with SRD by GPs with the same documentation in control patients. Moreover, it would also be interesting to compare the documentation of CVRFs in patients with SRD by GPs with the same data recorded by physicians other than GPs.

Regarding gender, bivariate analysis found that a greater number of CVRFs were recorded in women, coinciding with the results found by Baldiseroto et al. in the general population of Brazil [23]. However, Rahman et al. found no gender differences in a study conducted in the general population of Bangladesh [24]. Both bivariate analysis and the multivariate linear model found that the older the patient, the greater the number of registered CVRFs. This finding is consistent with the majority of studies performed in the general population [23-29]. In the bivariate analysis, it was shown that a greater number of CVRFs were recorded in patients who had a partner; however, these differences did not appear in the multivariate linear model. Baldiseroto et al. [23] found a higher prevalence of CVRFs in people with a partner; however, Rahman et al. [24] found that these people had the least prevalence. In two more studies that analysed this variable, no differences were found in the prevalence of CVRFs in the general population [28, 29]. Regarding educational level, only bivariate analysis showed significant differences, finding that patients with the highest educational level presented the least number of CVRFs. Comparing our results with other studies, Rahman et al.
[24] found no significant differences in the prevalence of CVRFs between different educational levels, however, the study carried out in Brazil by Baldiseroto et al. [23] found similar results to ours. Interestingly, there were several studies that analysed this variable by gender, finding a lower prevalence of CVRFs at higher educational levels, but only in women [26, 28-31]. The multivariate linear model showed that homeless patients had fewer CVRFs recorded than other patients. We did not find any study that compared this variable with the number of CVRFs. Bivariate analysis showed that, with respect to patients receiving welfare benefits, patients who were in other work situations (except housewives) had less CVRFs recorded. This result is similar to that found in a study carried out by Gazhali et al. in Malaysia, in which a greater number of CVRFs were identified among housewives [29]. This was due to the high prevalence of obesity, low fruit and vegetable consumption and physical inactivity [29]. Another study carried out in the Philippines found that the prevalence of CVRFs was highest among the self-employed and lowest among individuals with irregular employment [27]. This could be related to the fact that in the same study it was found that individuals with the highest level of education had the highest prevalence of CVRFs, and perhaps these individuals did not carry out irregular work. However, the multivariate linear model showed significant differences only in patients with temporary work disability compared to those who received welfare benefits or pensions. Contrary to our 
results, a study of the general population of Catalonia (Spain) identified no differences in the record of CVRFs in terms of patients' employment status [25]. In addition, more CVRFs were recorded in urban areas, similar to findings attained in the general Polish population [30] and in developing countries [27, 29]. By contrast, a study conducted in the USA by Trivedi et al. found a higher prevalence of metabolic syndrome in rural compared to urban settings [31]. Bivariate analysis showed a higher number of CVRFs in patients living within a socioeconomically deprived area and, regarding the global level of severity, the most severely affected patients had less CVRFs than the less severely affected. However, we did not find any study that compares these variables with the prevalence of CVRFs. There was no significant difference between ICD-10 codes for the number of recorded CVRFs. Finally, this model showed that more visits to primary care services were associated with an increased number of recorded CVRFs. This relationship seems logical because the more contact a patient has with health services, the greater the potential for their physical comorbidities to be recorded.

\section{Strengths and limitations of this study Limitations}

- This report was a cross-sectional study, from which we could only infer association and not causality.

- The study lacked a control group of patients selected from the same GP practices who were not diagnosed with significant mental illness.

- We were unable to obtain data on the prevalence of CVRFs in the general population. However, we obtained data on the prevalence of CVRFs in the general population of the same area in previous studies.

- Clinical diagnoses were not made through clinical interviews, but by the patients' long-term psychiatrists and updated in the RESMA database.

- We selected patients diagnosed with SRD and we have not taken into account the evolution and the course of the disorder. However, most of the patients who had CVRFs recorded had a stable course of the illness.

- Although almost all patients were being treated with antipsychotic medications, we did not take into account the dose and the type of drugs in the clinical variables analysed.

- Information about patients was collected from medical records instead of having individual patients undergo specific health screening tests.
- The study excluded patients who died during the follow-up. This may have led to an underestimation of the cardiovascular events in the study sample.

\section{Strengths}

- We analysed a large number of patients with SRD in a primary care setting.

- The study was performed in a wide catchment area, including 13 PCCs.

- This study represents information from real clinical practice.

\section{Conclusions}

This study demonstrated that some patients' demographic variables as well as use of primary care services were associated with the CVRF records compiled by patients' GPs. Given that patients with SMI have higher rates of mortality and morbidity from physical health problems compared to the general population, greater effort to document these risk factors in patients with SRD should be made by GPs.

\section{Abbreviations}

$\mathrm{Cl}$ : Confidence interval; CMU-MH: Clinical management unit of mental health; CVRFs: Cardiovascular risk factors; GLS: Global level of severity; GP: General practitioner; ICD: International classification of diseases; PCC: Primary care centre; RESMA: Malaga schizophrenia case registry; SMI: Severe mental illness; SRD: Schizophrenia and related disorders.

\section{Acknowledgements}

The authors acknowledge the Primary Care District of Malaga, particularly Maximiliano Vilaseca and Francisco Javier Navarro for their support. We are also grateful to the directors and general practitioners at all the general practices who participated in this study. Finally, we thank Santiago Galan for his assistance with information collection.

\section{Authors' contributions}

MCC, CMP, AGR and BMK contributed to conception and design, acquisition of data and analysis and interpretation of data. MCC, CMP and BMK were involved in drafting the manuscript or critically revising it for important intellectual content. All authors read and approved the final manuscript.

\section{Funding}

This project was funded by Consejería de Economía, Innovación, Ciencia y Empleo, Junta de Andalucía (Award Number: P10-CTS-5862, CTS-945) and Fundación Progreso y Salud (Award Number: PI-0193/2014).

\section{Availability of data and materials}

The data that support the findings of this study are available from Berta Moreno Küstner, but restrictions apply to the availability of these data, which were used under license for the current study, and so are not publicly available. Data are, however, available from the authors upon reasonable request and with permission of Berta Moreno Küstner.

\section{Ethics approval and consent to participate}

The authors assert that all procedures contributing to this work comply with the ethical standards of the relevant national and institutional committees on human experimentation and with the Helsinki Declaration of 1975, as revised 
in 2008. All procedures involving patients were approved by the Health Research Ethics Committee of the Health District of Malaga.

\section{Consent for publication}

Not applicable.

\section{Competing interests}

None.

\section{Author details}

${ }^{1}$ Department of Personality, Assessment and Psychological Treatment, Faculty of Psychology Andalusian Group of Psychosocial Research (GAP), University of Malaga, Campus Teatinos, 29071 Malaga, Spain. ${ }^{2}$ North East Granada Sanitary District, Clinical Management Unit at Marquesado, Andalusian Health Service, Carretera los Pozos, 18518 Alquife, Granada, Spain. ${ }^{3}$ Department of Health Economics and the Rational Drug Use of Medicines. Faculty of Medicine, University of Malaga, Campus Teatinos, 29071 Malaga, Spain. ${ }^{4}$ Clinical Management Unit of Mental Health of the Regional Hospital of Malaga. AndaIusian Health Service, Biomedical Research Institute of Malaga (IBIMA), Plaza del Hospital, 29009 Malaga, Spain. ${ }^{5}$ Department of Personality, Assessment and Psychological Treatment, Faculty of Psychology. Andalusian Group of Psychosocial Research (GAP). Biomedical Research Institute of Malaga (IBIMA), University of Malaga, Campus Teatinos, 29071 Malaga, Spain.

Received: 15 December 2019 Accepted: 10 May 2020

Published online: 19 May 2020

\section{References}

1. Temmingh HS, Oosthuizen PP. Pathways to care and treatment delays in first and multi episode psychosis. Soc Psychiatry Psychiatr Epidemiol. 2008;43:727-35.

2. Tosh G, Clifton A, Bachner MN. General physical health advice for people with serious mental illness. Schizophr Bull. 2011;37(4):671-3.

3. Smith DJ, Langan J, McLean G, Guthrie B, Mercer SW. Schizophrenia is associated with excess multiple physical-health comorbidities but low levels of recorded cardiovascular disease in primary care: cross-sectional study. BMJ Open. 2013;3(4):e002808.

4. Mitchell AJ, Vancampfort D, Sweers K, van Winkel R, Yu W, De Hert M. Prevalence of metabolic syndrome and metabolic abnormalities in schizophrenia and related disorders - a systematic review and metaanalysis. Schizophr Bull. 2013;39(2):306-18.

5. Osborn DP, Wright CA, Levy G, King MB, Deo R, Nazareth I. Relative risk of diabetes, dyslipidaemia, hypertension and the metabolic syndrome in people with severe mental illnesses: systematic review and metaanalysis. BMC Psychiatry. 2008;8:84

6. Pérez-Piñar M, Mathur R, Foguet Q, Ayis S, Robson J, Ayerbe L. Cardiovascular risk factors among patients with schizophrenia, bipolar, depressive, anxiety, and personality disorders. Eur Psychiatry. 2006;35:8-15.

7. Osborn DP, Nazareth I, King MB. Risk for coronary heart disease in people with severe mental illness-Cross-sectional comparative study in primary care. Br J Psychiatry. 2006;188:271-7.

8. Ferreira L, Belo A, Abreu-Lima C. A case-control study of cardiovascular risk factors and cardiovascular risk among patients with schizophrenia in a country in the low cardiovascular risk region of Europe. Rev Port Cardiol. 2010;29(10):1481-93.

9. Protopopova D, Masopust J, Maly R, Valis M, Bazant J. The prevalence of cardiometabolic risk factors and the ten-year risk of fatal cardiovascular events in patients with schizophrenia and related psychotic disorders. Psychiatr Danub. 2012;24(3):307-13.

10. Joshi KB, Nillawar A, Thorat AP. Cardiovascular disease risk in schizophrenia patients: a case control study. J Clin Diagn Res. 2013;7(12):2694-6.

11. Gardner-Sood P, Lally J, Smith S, Atakan Z, Ismail K, Greenwood KE, et al. Cardiovascular risk factors and metabolic syndrome in people with established psychotic illnesses: baseline data from the IMPaCT randomized controlled trial. Psychol Med. 2015;45:2619-29.

12. Oud MJT, Meyboom-de Jong B. Somatic diseases in patients with schizophrenia in general practice: their prevalence and health care. BMC Fam Pract. 2009;10:32.
13. Woodhead C, Ashworth M, Broadbent M, Callard F, Hotopf M, Schofield $P$, et al. Cardiovascular disease treatment among patients with severe mental illness: a data linkage study between primary and secondary care. Br J Gen Pract. 2016;66(647):e374-81.

14. Mitchell AJ, Lord O, Malone D. Differences in the prescribing of medication for physical disorders in individuals with $v$. without mental illness: meta-analysis. Br J Psychiatry. 2012;201:435-43.

15. Lester HE, Tritter JQ, Sorohan H. Patients' and health professionals' views on primary care for people with serious mental illness: focus group study. Br Med J. 2005;330(7500):1122-6.

16. Moreno-Küstner B, Mayoral F, Pérez O, Angona P, Requena J, García-Herrera JM, et al. The Malaga schizophrenia case-register (RESMA): overview of methodology and patient cohort. Int J Soc Psychiatry. 2009;55(1):5-15.

17. Castillejos MC, Martín-Pérez C, Carlos Mayoral F, Bordallo-Aragón A, Sepúlveda-Muñoz J, Moreno-Küstner B. Factors associated with visits to general practitioners in patients with schizophrenia in Malaga. BMC Fam Pract. 2018;19:180. https://doi.org/10.1186/s12875-018-0866-7.

18. Moreno-Küstner B, Mayoral F, Navas-Campaña D, García-Herrera JM, Angona P, Martín C, et al. Prevalence of schizophrenia and related disorders in Malaga (Spain): results using multiple clinical databases. Epidemiol Psychiatr Sci. 2016;25:38-48.

19. Moreno-Küstner B, Mayoral F, Rivas F, Angona P, Requena JM, GarcíaHerrera J, et al. Factors associated with use of community mental health services by schizophrenia patients using multilevel analysis. BMC Health Serv Res. 2011;11:257. https://doi.org/10.1186/1472-6963-11-257.

20. Valdés S, García-Torres F, Maldonado-Araque C, Goday A, Calle-Pascual A, Soriguer $F$, et al. Prevalence of obesity, diabetes and other cardiovascular risk factors in andalusia (Southern Spain). Comparison with national prevalence data. The Di@bet.es Study. Rev Esp Cardiol. 2014;67(6):442-8.

21. Viñas L, Fernández M, San Emeterio L, Luis M, Martín-López LM, Grup Psicosi Litoral. Detection of cardiovascular risk factors in patients with psychosis. Aten primaria. 2011;43(7):386-7.

22. Bernardo M, Cañas F, Banegas JR, Casademont J, Riesgo Y, Varela C, et al. Prevalence and awareness of cardiovascular risk factors in patients with schizophrenia: a cross-sectional study in a low cardiovascular disease risk geographical area. Eur Psychiatry. 2009;24(7):431-41.

23. Buckland G, Salas-Salvadó J, Roure E, Bulló M, Serra-Majem L. Sociodemographic risk factors associated with metabolic syndrome in a Mediterranean population. Public Health Nutr. 2008;11(12):1372-8.

24. Sygnowska E, Piwońska A, Waśkiewicz A, Broda G. Socioeconomic factors and the risk of metabolic syndrome in the adult Polish population: the WOBASZ study. Kardiol Pol. 2012;70(7):718-27.

25. Sy RG, Llanes EJB, Reganit PFM, Castillo-Carandang N, Punzalan FER, Sison OT, et al. Socio-demographic factors and the prevalence of metabolic syndrome among filipinos from the life care cohort. J Atheroscler Thromb. 2014;21:S9-17.

26. Vernay M, Salanave B, de Peretti C, Druet C, Malon A, Deschamps V, et al. Metabolic syndrome and socioeconomic status in France: the French Nutrition and Health Survey (ENNS, 2006-2007). Int J Public Health. 2013;58:855-64.

27. Ghazali SM, Seman Z, Kee Chee Cheong KC, Hock LK, Manickam M, Kuay $L$, et al. Sociodemographic factors associated with multiple cardiovascular risk factors among Malaysian adults. BMC Public Health. 2015;15:68.

28. Rahman M, Nakamura K, Seino K, Kizuki M. Sociodemographic factors and the risk of developing cardiovascular disease in Bangladesh. Prev Med. 2015;48(4):456.

29. Baldisserotto J, Kopittke L, Nedel FB, Takeda SP, Mendonça CS, Sirena SA, et al. Socio-demographic characteristics and prevalence of risk factors in a hypertensive and diabetics population: a cross-sectional study in primary healthcare in Brazil. BMC Public Health. 2016;16:573.

30. Kwaśniewska M, Kaleta D, Dziankowska-Zaborszczyk E, Drygas W. Healthy behaviours, life style patterns and sociodemographic determinants of the metabolic syndrome. Centr Eur J Public Health. 2009;17(1):14-9.

31. Trivedi T, Liu J, Probst JC, Martin AB. The Metabolic syndrome: are rural residents at increased risk? J Rural Health. 2013;29(2):188-97.

\section{Publisher's Note}

Springer Nature remains neutral with regard to jurisdictional claims in published maps and institutional affiliations. 